\title{
CONSIDERACIONES PARA LA PROVISIÓN DE SERVICIOS DE REGULACIÓN DE FERTILIDAD PARA MUJERES DURANTE LA PANDEMIA POR COVID-19
}

Considerations for the Provision of Fertility Regulation Services for Women During the Covid-19 Pandemic

Leonardo Cristian Reyes- Torres ${ }^{1}$, Claudio Enrique López- Labarca ${ }^{2}$

Autor correspondencia: Leonardo Cristian Reyes-Torres

Correo electrónico: leonardo.reyes@uda.cl.

1. Departamento de Obstetricia y Puericultura. Facultad de Ciencias de la Salud. Universidad de Atacama. Máster en Anticoncepción, Salud Sexual y Reproductiva. Magíster en Gestión de Atención Primaria en Salud. (Atacama, Chile)

2. Departamento de Obstetricia y Puericultura. Facultad de Ciencias de la Salud. Universidad de Atacama. Magíster en Salud Pública. (Atacama, Chile)

Fecha de recepción: 12/08/2020 Fecha aceptación: 03/06/2021 


\section{RESUMEN}

INTRODUCCIóN. La salud sexual y reproductiva (SSR) constituye un derecho humano universal. Debido a las consecuencias generadas por la pandemia del Virus SARS-COV-2 las prestaciones en salud sexual y reproductiva han sido postergadas en diferentes países. En Chile, debido a las medidas de distanciamiento social y reorganización de los servicios, se ha observado una pérdida en el acceso y continuidad de las prestaciones a causa de la reorientación de los recursos de atención primaria de salud. Las consecuencias de estas decisiones afectan a las prestaciones preventivas en SSR y descontinuación de los controles de anticoncepción. Esto último se torna más grave si se observa que este nivel resuelve el $95 \%$ de las prestaciones de anticoncepción de toda la red pública. OBJETIVOS. Describir las principales recomendaciones internacionales disponibles respecto del manejo de la anticoncepción en tiempos de pandemia y generar propuestas generales aplicables a nuestro país. MATERIAL Y MÉTODOS. Se realizó una revisión de literatura en Pubmed, Biblioteca Cochrane y sitios web de las principales sociedades científicas internacionales de habla hispana e inglesa. RESULTADOS. Se identificaron 6 documentos que sistematizan la información reciente y actualizada para que los países puedan enfrentar de la mejor manera las problemáticas en anticoncepción. Los resultados fueron organizados en: implementación de sistema de consultas no presenciales, sugerencias para el acceso a información/ servicios y manejo de los distintos tipos de anticoncepción. CONCLUSIONES. La adopción de la totalidad o algunas de las medidas propuestas supone que un sistema de salud pueda prevenir las consecuencias derivadas de la pérdida de acceso y continuidad en los servicios de anticoncepción y SSR.

Palabras clave: Anticoncepción; Salud sexual y reproductiva; SARS-COV-2; COVID-19; Servicios de salud.

\section{ABSTRACT}

INTRODUCTION. The sexual and reproductive health (SRH) constitutes a universal human right. Due the consequences generated by the pandemic Virus SARS-COV-2 sexual and reproductive health benefits have been deprioritized in different countries. In Chile, a loss in access and continuity of benefits has been observed due to the reorientation of primary health care resources. The consequences of these decisions affected the preventive benefits in SRH and the discontinuation of contraceptive controls. The latter becomes more serious if it is observed that this level solves $95 \%$ of the contraceptive benefits of the entire public network. OBJECTIVES. Describe the main international recommendations available regarding the management of contraception in pandemic times and generate proposals for our country.

MATERIAL AND METHODS. It was done a literature review in Pubmed, the Cochrane Library and the websites of the main international Spanish and English-speaking scientific societies. RESULTS. 6 documents were identified that systematize the recent and updated information so that the countries can face better the problems in contraception. The results were organized into the following categories: implementation of a non-face-to-face consultation system, suggestions for access to information / services and management of different types of contraception. CONCLUSIONS. The adoption of all or some of the proposed measures implies that the health system can prevent the consequences derived from the loss of access and continuity in contraceptive and SRH services.

Keywords: Contraception; Sexual and Reproductive Health; SARS-COV-2; COVID-19; Health services. 


\section{INTRODUCCIÓN}

Los derechos sexuales y reproductivos son considerados derechos humanos (ONU, 1994). Dentro de ellos, la salud sexual y reproductiva (SSR) tiene un rol fundamental como derecho humano y sus prestaciones son consideradas prestaciones esenciales en salud (OPS, 2020). En muchos países del mundo, debido a la pandemia provocada por el virus SARS-COV-2 se han redireccionado recursos y postergado las prestaciones en SSR y anticoncepción (UNFPA, 2020). Esta situación parece razonable cuando un Estado debe articular sus acciones para poder contener las primeras consecuencias de una pandemia global. Sin embargo, cuando estas medidas permanecen inalterables en el tiempo, la evidencia muestra que se generan otras consecuencias negativas en la salud general y la SSR, afectando principalmente a las mujeres más vulnerables de la población.

En Chile, un $80 \%$ de las mujeres se encuentran adscritas al seguro público de salud (Ministerio de desarrollo social, 2018). De este porcentaje, más de 1.500.000 millones de mujeres en el año 2018 se encontraban en control por anticoncepción en atención primaria de salud (DEIS, 2020), por lo que limitar el acceso y la continuidad de prestaciones de SSR y anticoncepción afectará directamente a las mujeres del grupo más vulnerable de nuestro país. Debido a que la actividad sexual no cesa e inclusive aumenta durante las pandemias (Yuksel and Ozgor, 2020), las consecuencias de esta discontinuidad de la atención pueden traer consigo un aumento de embarazos no deseados, del aborto inseguro y aumento de infecciones de transmisión sexual e infección por Virus de inmunodeficiencia adquirida humana (VIH). La falta acceso a prestaciones de SSR y las medidas de restricción de tránsito y cuarentenas también afecta en los indicadores de violencia de género, maltrato infantil y delitos sexuales (UNFPA, 2020).

Los Estados deben adoptar estrategias para contener los efectos negativos en la salud de la población y en el sistema sanitario que deja la pandemia y, por otro lado, asegurar la continuidad de las prestaciones en SSR y anticoncepción, ya que estos deben velar y ser garantes de los derechos sexuales y reproductivos de las personas.

El presente documento corresponde a una revisión de la literatura basada en sugerencias y recomendaciones de diferentes entidades y expertos que estaba disponible a la fecha de su elaboración, ya que la evidencia científica derivada de estudios observacionales o experimentales no había sido publicada hasta ese momento. El objetivo de este trabajo consiste en recopilar las principales recomendaciones internacionales 
respecto del manejo de la SSR y anticoncepción en tiempo de pandemia y generar propuestas para nuestro país.

\section{MATERIAL Y MÉTODOS}

Se realizó una búsqueda de artículos publicados en inglés y español en las bases de datos Pubmed y Biblioteca Cochrane utilizando las palabras clave: anticoncepción, salud sexual y reproductiva, SARS-COV-2, COVID-19, servicios de salud y sus respectivos conceptos en inglés según la terminología MESH, usando los operadores booleanos and/or. Los criterios de inclusión fueron: artículos originales full text, ensayos clínicos con asignación aleatoria, estudios de cohorte, casos y controles, editoriales, opiniones de expertos, comunicaciones a congresos. No se consideraron criterios de exclusión. Al tratarse de una temática emergente solo se obtuvieron 2 estudios en la primera búsqueda. Como consecuencia, se procedió a la búsqueda de documentos con las mismas especificaciones en el buscador Google Académico. Esta segunda acción dio como resultado 4 fuentes nuevas que direccionaron a los sitios web de las principales sociedades científicas internacionales de habla hispana e inglesa donde se encontraron documentos con posicionamiento y recomendaciones, los cuales fueron revisados a texto completo para preparar esta investigación y que se resumen en las tablas 1 y 2. Las recomendaciones obtenidas provenían de la Organización Mundial de la Salud (OMS), Organización Panamericana de la Salud (OPS), Sociedad Española de Contracepción (SEC), Sociedad Italiana de Contracepción y Facultad de Cuidados en Salud Sexual y Reproductiva del Real Colegio de Obstetricia y Ginecología del Reino Unido.

Tabla 1. Cuadro resultados de la búsqueda en bases de datos PubMed y Biblioteca Cochrane.

\begin{tabular}{|c|c|c|c|}
\hline $\begin{array}{c}\text { BASE } \\
\text { DATOS }\end{array}$ & $\begin{array}{l}\text { KEYWORDS } \\
\text { OPERADORES } \\
\text { BOLEANOS }\end{array}$ & CRITERIOS INCLUSIÓN & \multirow[t]{2}{*}{$\begin{array}{l}\text { ENCONTRADOS } \\
\text { E } \\
\text { INCLUIDOS }\end{array}$} \\
\hline $\begin{array}{l}\text { PubMed- } \\
\text { Biblioteca } \\
\text { Cochrane }\end{array}$ & $\begin{array}{l}\text { Contraception or } \\
\text { sexual and } \\
\text { Reproductive Health } \\
\text { and SARS-COV2 or } \\
\text { COVID-19 and } \\
\text { health services }\end{array}$ & $\begin{array}{l}\text { Tipos de estudio: originales full text, } \\
\text { ensayos clínicos asignación aleatoria, } \\
\text { estudios cohorte, casos y controles. } \\
\text { Editoriales, Opiniones de expertos, } \\
\text { Comunicaciones a congresos. Idioma: } \\
\text { inglés, español }\end{array}$ & \\
\hline \multicolumn{4}{|c|}{$\begin{array}{c}\text { RESULTADOS } \\
\text { Título, URL, Autor, lugar, año. }\end{array}$} \\
\hline \multicolumn{4}{|c|}{$\begin{array}{l}\text { 1) Contraception during Coronavirus-Covid } 19 \text { pandemic. Recommendations of the Board of the Italian Society } \\
\text { of Contraception, https://www.tandfonline.com/doi/abs/10.1080/13625187.2020.1766016?journalCode=iejc20, } \\
\text { Fruzzetti, F. et al, } 2020 \text {. }\end{array}$} \\
\hline \multicolumn{4}{|c|}{  } \\
\hline
\end{tabular}


Tabla 2. Cuadro resultados de la búsqueda en bases de datos Google Académico

\begin{tabular}{|c|c|c|c|c|c|}
\hline $\begin{array}{l}\text { BASE DE } \\
\text { DATOS }\end{array}$ & $\begin{array}{c}\text { KEYWORDS } \\
\text { OPERADORES } \\
\text { BOLEANOS }\end{array}$ & $\begin{array}{l}\text { CRITERIOS DE } \\
\text { INCLUSIÓN }\end{array}$ & $\begin{array}{c}\text { ART. } \\
\text { ENCONTRADOS }\end{array}$ & $\begin{array}{c}\text { ART. } \\
\text { INCLUIDOS }\end{array}$ & $\begin{array}{c}\text { ART. } \\
\text { EXCLUIDOS }\end{array}$ \\
\hline $\begin{array}{l}\text { Google } \\
\text { Académico }\end{array}$ & $\begin{array}{l}\text { Contraception or } \\
\text { sexual and } \\
\text { Reproductive } \\
\text { Health and SARS- } \\
\text { COV2 or COVID- } \\
19 \text { and health } \\
\text { services. }\end{array}$ & $\begin{array}{l}\text { Tipos de estudio: } \\
\text { artículos originales } \\
\text { full text, ensayos } \\
\text { clínicos con } \\
\text { asignación } \\
\text { aleatoria, estudios } \\
\text { de cohorte, casos y } \\
\text { controles, } \\
\text { editoriales, } \\
\text { Opiniones de } \\
\text { expertos, } \\
\text { Comunicaciones a } \\
\text { congresos. Idioma: } \\
\text { inglés, español }\end{array}$ & 4 & 4 & 0 \\
\hline
\end{tabular}

1) Posicionamiento de la Sociedad Española de Contracepción sobre la atención a la Salud sexual y reproductiva durante el estado de Alarma Sanitaria por la pandemia del COVID-19, https://www.redisser.org/Posicionamiento\%20SEC\%20-\%20Covid.pdf, Sociedad Española de Contracepción (SEC), Madrid, 2020.

2) Planificación familiar: Un manual mundial para proveedores. https://www.who.int/reproductivehealth/publications/family planning/9780978856304/es/, Facultad de Salud Pública Bloomberg de Johns Hopkins and Organización Panamericana de la Salud, Baltimore y Washington, D.C, 2019.

3) FSRH CEU recommendation on extended use of the etonogestrel implant and $52 \mathrm{mg}$ levonorgestrelreleasing intrauterine system during COVID restrictions, https://bit.ly/3gZ2zQi, The Faculty of Sexual and Reproductive Healthcare (FSRH) of the Royal College of Obstetricians and Gynecologists, London, 2020 .

4) Coronavirus disease (COVID-19): Contraception and family planning. https://bit.ly/3gPUZc9, OMS, Washington, 2020.

\section{RESULTADOS Y DISCUSIÓN}

Como se señaló en el apartado anterior, después de dos búsquedas se obtuvieron un total de 8 documentos que fueron revisados a texto completo. Tras dicha revisión se procedió a organizar la información en tres apartados: (1) recomendaciones para el paso de la atención presencial a la no presencial; (2) sugerencias para el acceso a la información y 
servicios de anticoncepción y planificación familiar; (3) y manejo de los métodos anticonceptivos en contexto de pandemia COVID-19 desagregado por tipo de método.

\section{Implementación de un sistema de atención/orientación no presencial}

A propósito de las medidas de distanciamiento social y reorganización de los servicios, se recomienda coordinar e implementar un mecanismo de consulta remota (telefónica o videollamada) con las usuarias, que permitirá conocer su motivo de consulta como, inicio de anticoncepción, cambio de método, recambio de método por caducidad, efectos secundarios con el método actual, entre otros (Sociedad Española de Contracepción, 2020), y de esta forma poder conducirla a través de un flujograma de atención local. El flujograma de atención local deberá estar contextualizado dentro de las directrices ministeriales que se entreguen en el ámbito de la atención en salud sexual y reproductiva en el contexto de la pandemia de la COVID-19. El flujograma de atención local debe ser elaborado por referentes técnicos con conocimiento de los recursos disponibles en la red asistencial local y además con manejo técnico de las prestaciones de salud sexual y reproductiva.

A pesar de las limitaciones que representa una consulta no presencial, puede permitir responder de manera parcial o completa a los requerimientos de las usuarias, evitando concurrencias innecesarias al centro de salud y dejando la atención presencial solo a casos estrictamente necesarios.

Se debe contemplar también el acceso a este tipo de consultas a mujeres que no tengan acceso a tecnología o presenten alguna dificultad física o cognitiva para poder concretar la atención a distancia.

Se recomienda reflejar en la historia clínica que se ha realizado la consulta online debido a la situación de alarma sanitaria y que la usuaria lo entiende y lo acepta (Sociedad Española de Contracepción, 2020)

\section{Sugerencias para encargados de programa y tomadores de decisiones con respecto al acceso a información y servicios de anticoncepción y planificación familiar (OMS, 2020)}

- Planificar y desarrollar estrategias innovadoras para garantizar que la mayor cantidad posible de personas pueda acceder a la información y a los métodos anticonceptivos durante este período. 
- Aumentar el uso de teléfonos móviles y tecnologías digitales para ayudar a las personas a tomar decisiones sobre qué métodos anticonceptivos usar y cómo se puede acceder a ellos.

- Permitir que los trabajadores de la salud brinden información y servicios anticonceptivos según las pautas nacionales en la mayor medida posible. Esto es particularmente importante cuando el embarazo plantea un alto riesgo para la salud.

- Ampliar la disponibilidad de servicios anticonceptivos (incluyendo información y métodos) a través de lugares que no sean centros de salud, como farmacias, plataformas en línea y otros puntos de venta. Esto puede ser con o sin receta, según las pautas nacionales y el método anticonceptivo.

- Garantizar el acceso a la anticoncepción de emergencia.

- Permitir el acceso a la anticoncepción para mujeres y niñas en los períodos inmediatos posparto y posaborto cuando puedan acceder a los servicios de salud.

- Aumentar el uso de telesalud para el asesoramiento y el intercambio de mensajes relacionados con el uso seguro y efectivo de la anticoncepción y para la selección e iniciación de anticonceptivos.

- Asegurar un inventario adecuado para evitar posibles desabastecimientos en todos los niveles del sistema de salud.

- Preparar avisos para los usuarios sobre cómo pueden acceder a información, servicios y suministros anticonceptivos.

- Monitorear el consumo de anticonceptivos en su área para identificar posibles dificultades y escasez.

- Aumentar la disponibilidad y el acceso a los anticonceptivos que pueden ser utilizados por el cliente sin el soporte del proveedor de servicios.

\section{Manejo de la anticoncepción en el contexto de pandemia por SARS-COV-2}

Los anticonceptivos corresponden a métodos o procedimientos que previenen un embarazo en mujeres sexualmente activas, ya sean ellas o sus parejas quienes los usen. En lo posible, los métodos anticonceptivos deben cumplir con algunos requisitos y características que permitan a las personas alcanzar sus metas reproductivas en forma 
efectiva y segura para su salud y de acuerdo con su situación de vida (Ministerio de Salud de Chile, 2018).

En el sistema público de salud chileno, existe una amplia gama de métodos anticonceptivos, entre los que se incluyen píldoras, inyectables, implantes subdérmicos, dispositivos intrauterinos con cobre y liberadores de hormonas, condones y esterilización quirúrgica.

Los métodos anticonceptivos miden su eficacia según la capacidad para prevenir el embarazo en el uso típico o habitual. Los anticonceptivos reversibles de acción prolongada (ARAP o LARC según sus siglas en inglés), que incluyen implantes subdérmicos y dispositivos intrauterinos, representan una excelente alternativa anticonceptiva, con alta eficacia anticonceptiva y seguridad.

Es importante considerar que no existe ningún método con eficacia anticonceptiva total o absoluta, por lo tanto, bajo ninguna circunstancia se puede garantizar o esperar la eficacia absoluta y/o total de un anticonceptivo, pudiendo siempre, a pesar del uso perfecto del método, estar la chance de producirse un embarazo. Esto es muy importante informarlo a las usuarias de anticonceptivos.

Los Criterios Médicos de Elegibilidad para el uso de Anticonceptivos (OMS, 2015), las Normas Nacionales sobre Regulación de Fertilidad (Ministerio de Salud de Chile, 2018), y el Manual Mundial para Proveedores de Planificación Familiar (Facultad de Salud Pública Bloomberg de Johns Hopkins and OPS, 2019) son documentos que deben tenerse presentes en estas circunstancias, además de otras publicaciones internacionales más actualizadas.

En el contexto de la pandemia, dependiendo de las disposiciones sanitarias que se puedan instruir y de acuerdo con las recomendaciones citadas, es importante tener presente algunas medidas que se podrían llevar a cabo:

\section{Anticoncepción hormonal combinada (AHC)}

Actualizar la historia clínica mediante entrevista remota (telefónica o vía online) y en caso de no existir cambios clínicos relevantes, renovar la prescripción hasta una nueva cita presencial.

Para las usuarias de AHC, podría ser razonable en estas circunstancias, permitir la prescripción remota por los próximos 6-12 meses sin volver a verificar el índice de masa corporal (IMC) y la presión arterial. 
El riesgo asociado al embarazo no planificado probablemente sea mayor al riesgo relacionado con el uso de la $\mathrm{AHC}$.

Si en el último control no fueron identificadas contraindicaciones, se podría considerar la provisión de un suministro adicional de AHC sin revisión del historial médico.

\section{Usuaria de anticoncepción combinada oral (ACO), Parchey Anillo vaginal en} mujeres Covid-19 positivas (Fruzzetti et al., 2020)

Pueden continuarse en mujeres asintomáticas Covid-19 positivas o caso leve.

Cuando la neumonía es grave y la inmovilización en la cama y el aumento del riesgo tromboembólico requieren suspender el tratamiento, se debe informar a las mujeres que es probable que ocurra la menstruación.

El reinicio de un anticonceptivo AHC se puede realizar inmediatamente después de la recuperación.

\section{Píldora de progestágeno solo (POP)}

Actualizar la historia clínica mediante entrevista online o telefónica y renovar la prescripción hasta nueva cita presencial.

Reforzar la educación con respecto a la ingesta en la hora indicada no superando las 3 horas de retraso en el caso de las píldoras de levonorgestrel y 12 horas en el caso de las píldoras de desogestrel.

No olvidar que las POP pueden ser utilizadas tanto por mujeres que están en periodo de lactancia como las que no, y desde el posparto inmediato.

La presión arterial sobre 160/100 es categoría 2 para su uso, por lo tanto podrían utilizar POP este grupo de mujeres.

\section{Usuaria de POP Covid-19 positiva (Fruzzetti et al., 2020)}

Pueden continuar el uso las mujeres positivas para Covid-19 tanto asintomático y sintomático.

En el caso de mujeres hospitalizadas que se les suspenda el anticonceptivo, deben ser informadas de que pueden menstruar. 
El reinicio de un POP se puede realizar de inmediato después de la recuperación. La eficacia no está garantizada para los primeros 7 días y en este período se debe usar un condón.

\section{Acetato de Medroxiprogesterona en Depósito (AMPD)}

Se recomienda cambiar a POP en caso de no poder acceder a personal de salud que pueda realizar el procedimiento inyectable (si una mujer no tiene contraindicaciones para AMPD, es probable que no tenga contraindicaciones para POP a menos que la absorción o la adherencia al uso sean una preocupación importante, por lo tanto no se necesitaría una evaluación presencial).

Para que la efectividad sea óptima, es importante administrar la inyección cada 3 meses (13 semanas). La inyección puede administrarse hasta con 4 semanas de retraso sin perder su acción ni requerir protección adicional en ese periodo (OPS, 2019).

En caso de contar con AMPD con sistema uniject, proporcionar dosis para la autoadministración a las mujeres que hayan sido instruidas para el procedimiento.

\section{Implante de etonogestrel (IMP-ENG)}

En las personas que requieren reemplazo de su ENG-IMP a los 3 años se les puede informar que su riesgo de embarazo es muy bajo durante el primer año de uso prolongado (hasta 4 años para ENG-IMP) (FSRH of the Royal College of Obstetricians and Gynecologists, 2020).

El reemplazo puede retrasarse hasta un año después del vencimiento para evitar riesgos innecesarios de transmisión del coronavirus debido al contacto con profesionales de la salud.

Se debe informar a las usuarias que nunca se puede garantizar la efectividad anticonceptiva absoluta de ningún método.

Después de 4 años completos de uso de ENG-IMP se debe aconsejar a todas las mujeres que usen condones además de agregar un POP (Thaxton and Lavelanet, 2018).

Dependiendo del contexto sanitario, se sugiere que los únicos motivos para concertar una cita presencial para extraer el implante en situación de confinamiento por la pandemia son el deseo de gestación y los efectos secundarios graves. 
Mujeres usuarias de implantes Covid-19 positivas (Fruzzetti et al., 2020)

Puede continuarse el método en mujeres positivas para Covid-19 asintomáticas y asintomáticas.

\section{Dispositivo intrauterino (DIU) liberador de Levonorgestrel de $52 \mathrm{mg}$}

El riesgo de embarazo con el uso extendido más allá del tiempo recomendado en ficha técnica (en el sexto año de uso) es muy bajo (FSRH of the Royal College of Obstetricians and Gynecologists, 2020).

Se puede retrasar el recambio del DIU para evitar la cita presencial en la situación actual. Se debe explicar a la usuaria que la eficacia anticonceptiva no puede garantizarse, pero probablemente es adecuada y que puede recomendar uso de píldora de solo gestágenos si no existen contraindicaciones para su uso ni problemas de absorción o adherencia hasta el momento de la cita presencial o el uso de condones.

Por encima del sexto año de uso se debe recomendar uso de píldora de solo gestágenos. También se puede recomendar el uso de doble método hasta cita presencial (FSRH of the Royal College of Obstetricians and Gynecologists, 2020).

\section{Otros DIUs hormonales (LNG 19,5 mg y LNG 13,5 mg)}

Se puede recomendar a las usuarias de estos DIUs utilizar píldora de solo gestágenos o condones a partir de la fecha de caducidad del DIU.

Se sugiere que los únicos motivos para concertar una cita presencial para extraer el DIU en este momento de confinamiento por la alarma sanitaria son el deseo de gestación, los efectos secundarios graves y los signos de infección.

\section{DIU de cobre (DIU-Cu)}

El uso de T380A podría ser efectivo por hasta 12 años (Facultad de Salud Pública Bloomberg de Johns Hopkins and Organización Panamericana de la Salud, 2019).

En mujeres $<40$ años portadoras de DIU de cobre se recomienda uso de POP o condones en el momento que se alcance la fecha de caducidad hasta la cita presencial.

Cuando el DIU se inserta en mujeres $>40$ años se mantiene la recomendación de retirarlo 1-2 años después de la menopausia

Se sugiere hacer retiro de DIU-Cu solo en caso de deseo de embarazo, tener signos significativos de infección o tener efectos secundarios adversos graves. 


\section{Anticoncepción en el posparto}

La urgencia sanitaria debido a la pandemia por SARS-COV-2 restringe el acceso a anticoncepción en los centros de atención primaria de salud. Por lo tanto, se recomienda proporcionar anticoncepción de alta eficacia a las puérperas antes del alta.

Según los Criterios médicos de elegibilidad de la OMS (2015), el uso antes de las 6 semanas posparto de implantes anticonceptivos y POP son categoría 2 (una condición en la que las ventajas de usar el método generalmente superan los riesgos teóricos o probados). En el caso de los dispositivos intrauterinos, la inserción antes de las 48 horas incluyendo la inserción inmediatamente después del alumbramiento es categoría 1 (una condición para la cual no hay restricciones para el uso del método anticonceptivo). Por lo anterior, resulta importante realizar las gestiones y articulaciones de la red de salud necesarias para poder brindar esta prestación antes del alta de las puérperas.

\section{Anticoncepción de emergencia (AE)}

Se debe garantizar el acceso de manera continua e inmediata a la anticoncepción de emergencia. Por lo anterior, se debe coordinar la disposición y acceso expedito a la $\mathrm{AE}$, además de hacer difusión a la comunidad de ello.

La AE con Levonorgestrel no tiene ninguna contraindicación, por lo tanto cualquier mujer puede ingerirla (OMS, 2015).

En conjunto con la $\mathrm{AE}$ se debe ofrecer un anticonceptivo de uso regular para su inicio al día siguiente, además de proporcionar condones.

\section{CONCLUSIONES}

La información y los servicios de anticoncepción y planificación familiar salvan vidas y son importantes en todo momento. La actividad sexual no cesa con la pandemia COVID19, por lo tanto es crucial garantizar que las personas puedan acceder a servicios e información basados en los derechos para iniciar y/o continuar el uso de anticonceptivos.

La anticoncepción, además de ayudar a prevenir los embarazos no planificados y sus consecuencias negativas para la salud de niñas y mujeres (muerte, aborto inseguro, infecciones de transmisión sexual, entre otras) puede ayudar a evitar la presión que surgiría a partir de estos embarazos en los sistemas de atención sanitaria que están trabajando arduamente para abordar la pandemia por COVID-19. 
El presente estudio identificó ciertas recomendaciones que pueden ser adecuadas de implementar en nuestro país, con el fin de mantener la continuidad de los servicios de anticoncepción y SSR en concordancia con el combate de la pandemia.

1. Es deseable implementar un sistema de atención/orientación no presencial de manera remota, que no arriesgue innecesariamente a las usuarias a asistir a sus controles de anticoncepción.

2. Los casos en que el riesgo de asistir sea menor al riesgo de quedar sin protección anticonceptiva, deben ser atendidos de manera presencial tomando todas las medidas de protección indicadas por la autoridad respectiva.

3. Los tomadores de decisiones y niveles de gestión estratégica deben preparar este soporte de prestaciones (remota o presenciales) considerando el acceso permanente a la información, la coordinación de establecimientos de la red de salud y la disponibilidad de stock de anticonceptivos para asegurar la continuidad hacia las usuarias.

4. En relación con el manejo de los diferentes anticonceptivos en el periodo de pandemia, no han cambiado las indicaciones y contraindicaciones para su uso. Según la evidencia disponible en la actualidad, en el caso de usuarias con COVID-19 positivo con complicaciones severas del cuadro, se hace razonable la suspensión de la anticoncepción hormonal combinada por el aumento del riesgo de trombosis. 


\section{BIBLIOGRAFÍA}

Departamento de Estadísticas e Información de SAlud (DEIS). [Página web].

Disponible en: https://deis.minsal.cl/, 2020.

Facultad de Salud Pública Bloomberg De Johns Hopkins y ORganización

PANAMERICANA DE LA SALUD. Planificación familiar: Un manual mundial para proveedores. Baltimore y Washington, D. C: OPS, 2019.

Fondo de Población de las Naciones Unidas (UNFPA). Atención en Salud Sexual y Reproductiva y Planificación Familiar durante la emergencia sanitaria por la COVID-19Atención en Salud Sexual y Reproductiva y Planificación Familiar durante la emergencia sanitaria por la COVID-19. UNFPA, 2020, disponible en: https://lac.unfpa.org/sites/default/files/pub-pdf/3-Covid-SSRyPF_ESP\%20(1).pdf

Fruzzetti, F., et al. Contraception during Coronavirus-Covid 19 Pandemic. Recommendations of the Board of the Italian Society of Contraception. En: The European Journal of Contraception \& Reproductive Health Care. 2020, vol. 25, núm. 3, pp. 1-3.

Ministerio De Desarrollo Social. Encuesta CASEN 2017. Santiago de Chile: Ministerio de Desarrollo Social, 2018.

Ministerio De SAlud De Chile. Normas Nacionales Sobre Regulación de la Fertilidad. Santiago de Chile: MINSAL, 2018.

ORgANizACIÓN MUNDIAL DE LA SALUd (OMS). Medical eligibility criteria for contraceptive use Ginebra: OMS, 2015.

Organización Mundial de la SALud (OMS). Coronavirus disease (COVID-19): Contraception and family planning. OMS, 2020. Disponible en:

https://www.who.int/news-room/q-a-detail/coronavirus-disease-covid-19contraception-and-family-planning

ORgANIZACIÓN DE LAS NACIONES UNIDAS (ONU). Informe de la Conferencia Internacional sobre la Población y el Desarrollo. El Cairo: ONU, 1994.

ORGANIZACIÓN PANAMERICANA DE LA SALUD (OPS). La salud sexual y reproductiva en tiempos de COVID-19: Evidencias, perspectiva y desafios en América Latina y el Caribe. [Seminario web]. 3 de abril de 2020. Disponible en:

https://www.campusvirtualsp.org/es/webinar/la-salud-sexual-y-reproductiva-entiempos-de-covid-19-evidencias-perspectiva-y-desafios-en

Sociedad Española de ConTRACEPCiÓn. Posicionamiento de la Sociedad Española de Contracepción sobre la atención a la Salud sexual y reproductiva durante el estado de Alarma Sanitaria por la pandemia del COVID-19. Madrid: SEC, 2020.

Thaxton, L.; LAVElanet, A. Systematic review of efficacy with extending contraceptive implant duration. International Journal of Gynecology \& Obstetrics. 2018, vol. 144, núm. 1, pp. 2-8. 
The Faculty of Sexual and Reproductive Healthcare (Fsrh) of the Royal COLLEGE OF OBSTETRICIANS AND GYNECOLOGISTS. FSRH CEU recommendation on extended use of the etonogestrel implant and $52 \mathrm{mg}$ levonorgestrel-releasing intrauterine system during COVID restrictions. Londres: FSRH, 2020.

YUKSEL, B.; OzGOR, F. Effect of the COVID-19 pandemic on female sexual behavior. En: International Journal of Gynecology \& Obstetrics. 2020, vol. 150, núm. 1, pp. 98-102. 